\title{
Flake graphite cast iron investigated by a magnetic method
}

\author{
G. Vértesy ${ }^{1}$, T. Uchimoto ${ }^{2}$, T. Takagi ${ }^{2}$ and I. Tomáǵ \\ ${ }^{1}$ Research Centre for Natural Sciences, Institute of Technical Physics and Materials Science, \\ Budapest, H-1525 Budapest, P.O.B. 49, Hungary \\ ${ }^{2}$ Institute of Fluid Science, Tohoku University, 2-1-1 Katahira, Aoba-ku, Sendai, 980-8577, Japan \\ ${ }^{3}$ Institute of Physics, Academy of Sciences of the Czech Republic, Na Slovance 2, 18221 Praha, Czech Republic
}

\begin{abstract}
The method of Magnetic Adaptive Testing (MAT) was applied for investigation of flake graphite cast iron samples having various metallic matrix and graphite structure. MAT is typical by its low required magnetization of samples, because it is based on measurement of families of minor magnetic hysteresis loops. The flat samples were magnetized by an attached yoke and sensitive descriptors of their magnetic/structural state were obtained from evaluation of the measured data. Metallographic examination of the matrix and graphite structures was performed and results of the non-destructive magnetic tests were compared with these data. A very good correlation was found between the magnetic descriptors and the graphite morphology. Based on these results, MAT is suggested as a highly promising non-destructive alternative of destructive tests for monitoring structural changes in cast iron and in other ferromagnetic objects.
\end{abstract}

Index Termsठ Magnetic NDE, Magnetic adaptive testing, Cast iron

\section{INTRODUCTION}

Cast iron is one of the most frequently used industrial construction materials. Low cost of production, good machinability, and excellent possibilities of shaping the details by casting attract an intense interest of industry. The cast irons are generally many-component alloys of iron with large content of carbon. The cast iron structure is classified by its metallic matrix composition (ferrite, pearlite, carbides, etc.) and by morphology of its graphite inclusion. The mechanical properties are fundamentally dependent both on the matrix composition and on the graphite shape (flaky, spheroidal, vermicular, etc.), size and density [1]. One of the types of cast iron - the flake graphite cast iron - is frequently used for mechanical components in bearings, brake shoes, etc. because of its high wear resistance and damping capacity. The flake graphite cast iron is an ideal material for automobile brake disks since it has excellent damping properties and thermal conductivity just because of the flaky graphite.

An easy nondestructive check-up of properties of the graphite inclusions and of constituents of the cast iron matrices is highly desired. A basic ultrasonic technique evaluating the structure of graphite has been established, see e.g. [2]. However, this nondestructive method requires two parallel planes for each target specimen to evaluate the ultrasonic velocity, and it can only be applied with difficulty to specimens with a complicated outline. Measurement of electromagnetic properties such as conductivity and permeability [3] can also provide useful information. Some works have been carried out to estimate the hardness and the fraction of ferrite to pearlite in ductile cast iron using electromagnetic nondestructive evaluation methods. It has been reported that the eddy current method is highly accurate

Corresponding author: G. Vértesy (e-mail: vertesy.gabor@ttk.mta.hu). for the evaluation of ductile cast iron matrices [4,5]. The graphite flakes in flake graphite cast iron have various morphology since the flakes have complicated structures and are linked to each other. The relationship between the graphite structure and its electromagnetic properties was systematically investigated in [6].

Magnetic measurements are frequently used for characterization of changes in ferromagnetic materials, because magnetization processes are closely related to their microstructure. This makes the magnetic approach an obvious candidate for non-destructive testing, for detection and characterization of any defects in materials and in products made of such materials [7-10]. Structural non-magnetic properties of ferromagnetic materials have been nondestructively tested using traditional hysteresis methods since long time with fair success. A number of techniques have been suggested, developed and currently used in industry, see e.g. [11]. They are mostly based on detection of structural variations via the classical parameters of major hysteresis loops.

An alternative, more sensitive and more experimentally friendly approach to this topic was considered recently, based on magnetic minor loops measurement. The survey of this technique can be found in [12]. The method, called Magnetic Adaptive Testing (MAT) was presented, which introduced general magnetic descriptors to diverse variations in non-magnetic properties of ferromagnetic materials, optimally adapted to the just investigated property and material. MAT was successfully applied for characterization of material degradation in different specimens and it seems to be an effective tool e.g. for replacement of the destructive hardness and/or ductile-brittle transition temperature measurements.

In our previous works [13-15] magnetic characteristic parameters from a system of minor loops measured on a series of ductile cast iron samples were analyzed, and their sensitivity was evaluated. The purpose of the present work is 
to investigate flake graphite cast iron samples by MAT, and to find correlation between magnetic parameters and graphite morphology of the material.

\section{SAMPLE PREPARATION}

Three flake graphite cast iron materials with chemical compositions listed in Table 1 were prepared.

\section{TABLE 1 HERE}

Their carbon equivalent (CE) values were defined by:

$$
C E=\operatorname{mass} \% \mathrm{C}+\frac{1}{3}(\operatorname{mass} \% \mathrm{Si}+\operatorname{mass} \% \mathrm{P})
$$

and were controlled to produce various graphite shapes and sizes. These materials were designated as CE4.7, CE4.1 and CE3.7 based on their targeted CE values. Pig iron $(4.09 \% \mathrm{C}$, $0.89 \% \mathrm{Si}, \quad 0.07 \% \mathrm{Mn}, \quad 0.019 \% \mathrm{P}, \quad 0.012 \% \mathrm{~S}, \quad 0.016 \% \mathrm{Cr}$, $0.003 \% \mathrm{Ti})$, ferrosilicon $(\mathrm{Fe}-75 \% \mathrm{Si})$, electrolytic iron and electrolytic manganese were used as raw materials and were melted using a high frequency induction melting furnace at $1743 \mathrm{~K}$. Ferrosilicon $(\mathrm{Fe}-75 \% \mathrm{Si})$ was also used as an inoculant. The melts were poured into moulds made by the $\mathrm{CO}_{2}$ gas process to produce the columnar bars with length $60 \mathrm{~mm}$ and diameter $46 \mathrm{~mm}$. After that each bar was cut into disks $10 \mathrm{~mm}$ thick.

\section{Magnetic Adaptive Testing}

MAT investigates a complex set of minor hysteresis loops (from a minimum amplitude of the magnetizing field, with increasing amplitude by regular steps) for each sample of the measured series. It follows from the theory of Preisach model of hysteresis [16], that such a set of experimental data contains complex information on hysteresis of the measured material.

A specially designed Permeameter [17] with a magnetizing yoke was applied for measurement of families of minor loops of the magnetic circuit differential permeability. Size of the yoke was chosen to fit geometry of the samples: it was a C-shaped laminated $\mathrm{Fe}-\mathrm{Si}$ transformer core with crosssection $S=10 \times 5 \mathrm{~mm}^{2}$, the total outside length $18 \mathrm{~mm}$, and the total outside height of the bow $22 \mathrm{~mm}$. The magnetizing coil was wound on the bow of the yoke, with $N=200$ turns and the pick-up coil was wound on one of the yoke legs with $n=75$ turns.

The magnetizing coil gets a triangular waveform current with step-wise increasing amplitudes and with a fixed slope magnitude in all the triangles. This produces a triangular time-variation of the effective field in the magnetizing circuit and a signal is induced in the pick-up coil. As long as the field sweeps linearly with time, the voltage signal in the pick-up coil is proportional to the differential permeability of the magnetic circuit. The Permeameter works under full control of a PC computer, which registers data-files for each measured family of the minor ñpermeability loopsò.
The experimental raw data are processed by an evaluation program, which divides the originally continuous signal of each measured sample into a family of individual permeability half-loops. The program filters experimental noise and interpolates the experimental data into a regular square grid of elements, $\mu_{i j} \equiv \mu\left(h_{a i}, h_{b j}\right)$, of a $\mu$-matrix with a pre-selected field-step. The coordinates $h_{a i}, h_{b j}$ of the elements represent the actual magnetic field value, $h_{a i}$, on the actual minor loop with amplitude $h_{b j}$. Each $\mu_{i j}$-element represents one ñMAT-descriptorò of the investigated material structure variation.

The matrices are processed by another evaluation program, which normalizes them by a chosen reference matrix, and arranges each set of the mutually corresponding elements $\mu_{i j}$ of all the evaluated $\mu$-matrices into a $\mu_{i j}(x)$ degradation function. Here $x$ can be any independently measured parameter. In our case this is either area or length of the graphite flakes, determined independently in the samples. For details of the whole MAT procedure see [12].

\section{Results}

The metallographic examination of the matrix and graphite structures were done according to ISO 945. Microphotographs (shown in Fig. 1) of the three materials in their as-cast condition revealed that the graphite

\section{FIG. 1 HERE}

flakes of CE4.7 are relatively long, they are uniformly and isotropically distributed, and are thus categorized as type-B flakes defined by ISO 945. CE4.1 has smaller graphite flakes than CE4.7 and they are categorized as type-A flakes. In CE3.7 very small eutectic graphite flakes were found to be distributed in the dendrite and they are categorized as type-D and type-E flakes. Microphotographs of the samples after etching with $3 \%$ Nital indicated that CE4.7 had a pearliteferrite matrix, CE3.7 had a completely pearlitic matrix, and CE4.1 mainly had a pearlitic matrix but a small amount of ferrite surrounded the graphite flakes.

The area fraction and the average length of the graphite flakes were evaluated using an image processing software. The area fraction of graphite was evaluated using microphotograph binary images of 5 sample regions at the same magnification as that in Fig. 1. The length of graphite is defined as the average diameter of the minimum circle circumscribing each graphite flake larger than $5 \mu \mathrm{m}$. The area fraction of graphite for CE4.7, CE4.1 and CE3.7 is 17.8, 12.6 and $10.0 \%$, respectively. The length of the graphite flakes for CE4.7, CE4.1 and CE3.7 is 67, 39 and $28 \mu \mathrm{m}$, respectively.

MAT measurements were performed on each sample and the MAT descriptors were evaluated. The optimal MAT degradation functions (the $\mu$-degradation functions) were considered as functions of the given independent parameters, e.g. the length of the graphite flakes and the area fraction of graphite. Note that the shown MAT degradation functions are 
normalized by the corresponding value of the sample having the smallest graphite area and length (CE3.7).

The correlation between magnetic parameters and graphite area can be seen in Fig. 2, while correlation between magnetic parameters and graphite length can be seen in Fig. 3.

\section{FIG. 2 HERE}

\section{FIG. 3 HERE}

\section{DISCUSSION}

In construction materials, made of flake graphite cast iron, the graphite morphology plays an important role in mechanical behavior of the material. Material process condition (chemical component, cooling rate, pouring temperature, etc.) modifies the graphite morphology.

Metallurgical tests are therefore very important, and they should be performed in many cases. However, these tests are rather complicated, time consuming and destructive. Any nondestructive method, which can replace this destructive one, would be very helpful.

The graphite morphology influences also magnetic behavior of the flake graphite cast iron. It is evident from the above presented graphs (Figs. 2 and 3) that closely linear correlation was found between geometric values of the graphite flakes and the MAT parameters. These magnetic parameters are sensitive enough and can be measured easily and non-destructively.

The MAT method gives multi-parametric results by its principle and the matrix-evaluation program calculates sensitivity of each degradation function and draws their ñsensitivity mapò in the plane of the field coordinates $\left(h_{a i}, h_{b j}\right) \equiv(i, j)$. This map shows the relative sensitivity of each $\mu_{i j}(x)$-degradation function with respect to the independently measured, $x$, of the investigated material. Sensitivity of each degradation function is computed as the slope of its linear regression and it is expressed by a color and/or shade in the sensitivity map figure. The sensitivity maps help to choose the most sensitive and at the same time the most reliable (the most reproducible) degradation functions.

Fig. 4 shows the sensitivity map of the $\mu_{i j}$ (graphite length)-degradation functions. The crossing lines show the point, where the degradation function of Fig. 3 was taken from. (The sensitivity map is very similar, if the graphite area is considered.) It is seen, that these degradation functions were taken from the top sensitivity area, which appears in the graph in white. There is a plateau around this point, which ensures reliability (and reproducibility) of the points: if the measurement is repeated and the new parameter is taken not exactly from the center of this area, the evaluated MAT degradation functions are not modified (if they remain within the relatively large white area).

The samples are magnetized during the measurement by a magnetizing yoke, which is placed on the flat surface of the sample. This experimental arrangement means an open magnetic circuit, because some magnetic flux is always scattered at the air gap between the yoke and the sample. To get reliable MAT-data, quality of the surface must not vary from sample to sample and conditions of the measurement must be kept constant within the each series of experiments. The exact value of the magnetic field inside the sample is not known/measured in the used experimental arrangement. Because of this, instead of the magnetic field (given in $\mathrm{A} / \mathrm{m}$ ), the value of the magnetizing current (given in $\mathrm{mA}$ ) is used as $h_{a i}$ and $h_{b j}$ when the $\mu_{i j} \equiv \mu\left(h_{a i}, h_{b j}\right)$ matrix elements are given in Fig. 4.

The poor repeatability due to the uncertainty of the value of the parasitic air-gap between sample and yoke would be solved if the field inside the sample were measured instead of the magnetizing current. In this case the measuring/evaluating set-up would be more complicated, as the only adequate method would be to measure the surface field by a cascade of Hall probes and to extrapolate the fielddata down to the sample surface, as suggested in [18]. A less scientific but more technical/practical approach, is to decrease the relative air-gap fluctuation by application of an artificial non-magnetic spacer between the sample and the yoke surfaces. The resulting scatter of the MAT-data (while using the magnetizing current approach) is then comparable to the field-measuring approach, but the measuring/evaluating setup is substantially simpler [19]. However, fortunately, in the present case the surfaces of the measured samples were rather smooth and homogenous, which made the measurement very much repeatable without applying the above mentioned techniques.

\section{CONCLUSIONS}

The method Magnetic Adaptive Testing, which is based on nondestructive, systematic measurement of minor magnetic hysteresis loops was applied for three flake graphite cast iron materials with different chemical compositions and different matrix and flake graphite properties. MAT was shown to be a useful tool for finding correlation between the chosen nondestructively measured magnetic parameters and the graphite morphology. Linear correlations with very small scatter of points were found between the optimally chosen MAT degradation functions and both the graphite length and the graphite area of the as-cast samples.

We may conclude, that Magnetic Adaptive Testing is an experimentally friendly and sensitive method for tests of the cast iron structure. Through its multi-parametric principle MAT (i) contributes to solving the problem of similar magnetic manifestations of unlike material causes. By its use of minor magnetizing loops MAT (ii) suppresses the requirement of the material magnetic saturation, while at the same time (iii) it exceeds sensitivity of the traditional magnetic tests.

\section{ACKNOWLEDGMENT}

This work was partly supported by the JSPS Core-to-Core Program, AAAdvanced Research Networksò (International research core on smart layered materials and structures for 
energy saving). One of the co-authors (I.T.) appreciates financial support by the project No.TA02011179 of the Technical Agency of the Czech Republic.

\section{REFERENCES}

[1] C.F. Walton and T.J. Opar (Eds.), Iron casting handbook, Iron Casting Society, Inc., New York, 1981

[2] T. Abe and K. Ikawa : IMONO 58 (1986) 479.

[3] A.B. Everst: Foundry Trade J., 89 (1950) 57.

[4] T. Uchimoto, T. Takagi, S. Konoplyuk, T. Abe, H. Huang and M. Kurosawa, Eddy current evaluation of cast iron for material characterization, J. Magn. Magn. Mater., 258-259 (2003) 493.

[5] S. Konoplyuk, T. Abe, T. Uchimoto, T. Takagi and M. Kurosawa, Characterization of ductile cast iron by eddy current method, NDT\&E Int., 38 (2005) 623

[6] T. Uchimoto, T. Takagi, T. Abe, Electromagnetic nondestructive evaluation of graphite structures in flake graphite cast iron, Materials Transactions, 51 (2010) 1114.

[7] H. Kronmüller and M. Fähnle, Micromagnetism and the Microstructure of Ferromagnetic Solids, Cambridge University Press, Cambridge, 2003.

[8] D.C. Jiles, Magnetic methods in nondestructive testing, K.H.J. Buschow et al., Ed., Encyclopedia of Materials Science and Technology, Elsevier Press, Oxford, p.6021, 2001

[9] I. Mészáros, Complex magnetic investigation of ferritic stainless steel, Materials Science Forum, vol. 473-474, p. 231, 2005.

[10] D.C. Jiles, Review of magnetic methods for nondestructive evaluation, NDT International, vol. 21, p.311, 1988 .

[11] J. Blitz, Electrical and magnetic methods of nondestructive testing, Bristol, Adam Hilger IOP Publishing, Ltd., 1991.

[12] I.Tomág் G.Vértesy, ĂMagnetic Adaptive Testingñ in Nondestructive Testing Methods and New Applications, M.Omar (Ed.), ISBN: 978-95351-0108-6, (2012), InTech: http://www.intechopen.com/articles/show/title/magnetic-adaptive-testing.

[13] G. Vértesy, T. Uchimoto, T. Takagi, I. Tomáġ O. Stupakov, I. Meszaros, J. Pavo, Minor hysteresis loops measurements for characterization of cast iron Physica B, 372 (2006), pp. 156-159

[14] G. Vértesy, T. Uchimoto, I. Tomág T. Takagi, Nondestructive characterization of ductile cast iron by Magnetic Adaptive Testing, J.Magn.Magn.Mater. 322 (2010) 3117-3121.

[15] I. Tomáġ B. Skrbek, T. Uchimoto, J. Kadlecová, O. Stupakov, O. Perevertov, J. Dol ekal, Application of Magnetic Adaptive Testing to Cast Iron, Acta Metallurgica Slovaca, 13 (2007) pp.129 ï 132.

[16] I.D. Mayergoyz, Mathematical models of hysteresis, Springer-Verlag, New York, 1991

[17] I. Tomág் and O. Perevertov, Permeameter for Preisach approach to materials testing, JSAEM Studies in Applied Electromagnetics and Mechanics 9, ed. T.Takagi and M.Ueasaka, IOS Press, Amsterdam, p. 5., 2001.

[18] O. Stupakov, System for controllable magnetic measurement with direct field determination, J. Magn. Magn. Mater. 324 (2012) pp. 631-636.

[19] I. Tomáġ J. Kadlecová, G. Vértesy, Measurement of flat samples with rough surfaces by Magnetic Adaptive Testing, IEEE Trans Magn, 48 (2012) pp. 1441-1444.

TABLE I

CHEMICAL COMPOSITION OF THE FLAKE GRAPHITE CAST IRON SAMPLES (VALUES IN WT\%)

\begin{tabular}{|c|c|c|c|c|c|c|c|c|}
\hline \multirow{2}{*}{ Sample } & \multicolumn{7}{|c|}{ Chemical composition } & \multirow{2}{*}{$\begin{array}{c}\text { CE } \\
\end{array}$} \\
\cline { 2 - 10 } & $\mathrm{C}$ & $\mathrm{Si}$ & $\mathrm{Mn}$ & $\mathrm{P}$ & $\mathrm{S}$ & $\mathrm{Cr}$ & $\mathrm{Ti}$ & $(\%)$ \\
\hline CE4.7 & 3.77 & 2.78 & 0.78 & 0.025 & 0.015 & 0.029 & 0.015 & 4.71 \\
\hline CE4.1 & 3.36 & 2.15 & 0.69 & 0.018 & 0.010 & 0.014 & 0.011 & 4.08 \\
\hline CE3.7 & 3.13 & 1.66 & 0.72 & 0.017 & 0.020 & 0.038 & 0.010 & 3.69 \\
\hline
\end{tabular}
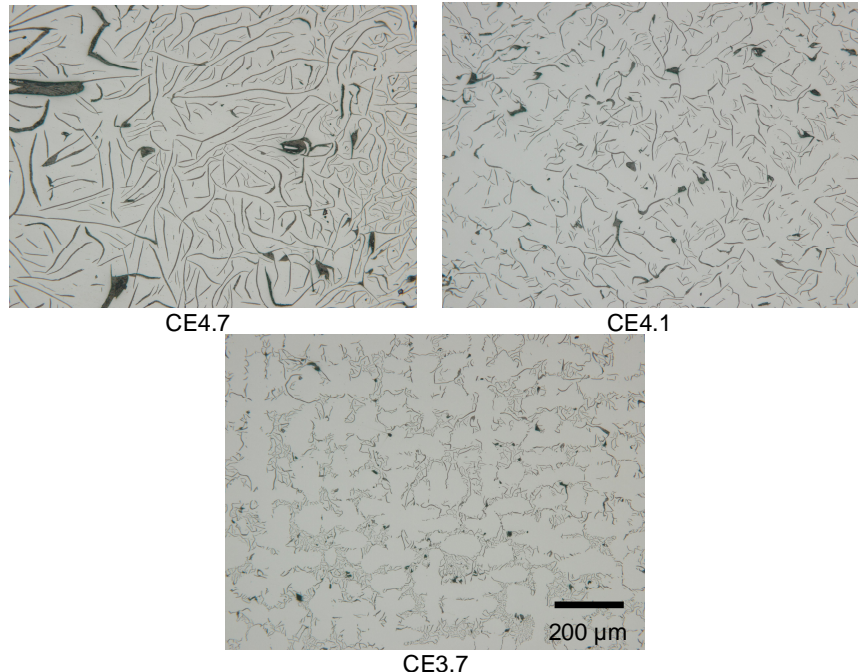

CE4.1

CE3.7

Fig. 2. The optimally chosen MAT descriptor vs. graphite area of the investigated samples - the optimal $\mu_{i j}$ (graphite area)-degradation function.

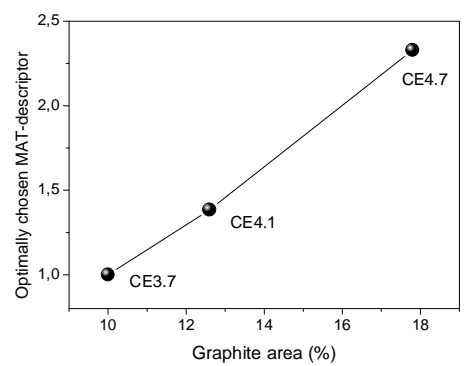

Fig. 3. The optimally chosen MAT descriptor vs. graphite length of the investigated samples - the optimal $\mu_{i j}$ (graphite length)-degradation function.

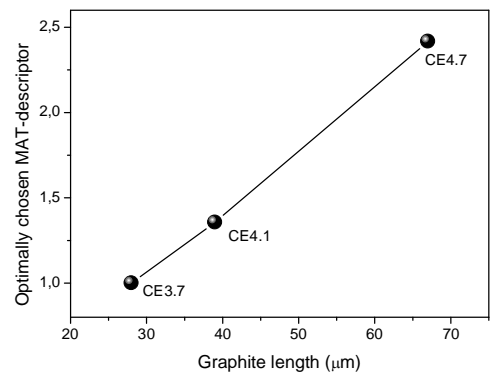

Fig. 4. Map of relative sensitivity of the $\mu_{i j}$ (graphite length)-degradation functions. The crossing lines show the point, from where descriptors of Fig. 3 were taken.

Fig. 1. Microphotographs of the three investigated materials 


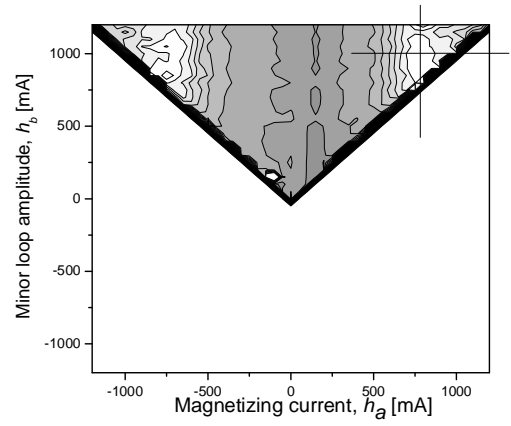

